\title{
The Efficacy of N-Butylphthalide and Dexamethasone Combined with Hyperbaric Oxygen on Delayed Encephalopathy After Acute Carbon Monoxide Poisoning
}

This article was published in the following Dove Press journal:

Drug Design, Development and Therapy

\section{Jiefang Zhang (D) \\ Yuewen Guo ${ }^{2}$ \\ Wenyao $\mathrm{Li}^{1}$ \\ Guangli $\mathrm{Li}^{3}$ \\ Yankun Chen (iD) 4}

'Intensive Care Unit, Heze Municipal Hospital, Heze City, Shandong Province, People's Republic of China; ${ }^{2}$ Emergency Department, Heze Municipal Hospital, Heze City, Shandong Province, People's Republic of China; ${ }^{3}$ Department of Pharmacy, Heze Municipal Hospital, Heze City, Shandong Province, People's Republic of China; ${ }^{4}$ Department of Neurology, Heze Municipal Hospital, Heze City, Shandong Province, People's Republic of China
Correspondence: Yankun Chen Department of Neurology, Heze Municipal Hospital, 2888 Caozhou Road, Heze City 274000, Shandong Province, People's Republic of China Email kundan@I26.com

\begin{abstract}
Background: Carbon monoxide (CO) poisoning is a common health problem among people in many countries, primarily because of its severe clinical effects and high toxicological morbidity and mortality. Acute brain injury and delayed encephalopathy after acute carbon monoxide poisoning (DEACMP) are the most common neurological complications. This study was performed to assess the efficacy of N-butylphthalide (NBP) and dexamethasone (DXM) combined with hyperbaric oxygen (HBO) in patients with DEACMP.

Patients and Methods: A total of 171 patients with DEACMP were recruited and assigned to the combined therapy group (receiving NBP and DXM $5 \mathrm{mg}$ /day plus HBO therapy) or the control group (HBO therapy as monotherapy). Conventional treatments were provided for all patients. The cognition and movement changes in patients were evaluated by the MiniMental State Examination (MMSE), the Montreal Cognitive Assessment (MoCA) scale and the Barthel index of activities of daily living (ADL) before and after the treatment at 1 month, 3 months, and 1 year, respectively.
\end{abstract}

Results: At 1 month, 3 months, and 1 year after the treatment, the MMSE, MoCA and ADL scores were all significantly higher in the combined therapy group than those in the control group. There were no significant alterations in blood glucose, blood lipids, or liver and kidney function during the whole treatment session. Some patients experienced loss of appetite, mild headache and minor skin irritations. However, these patients recovered by themselves and needed no additional medications or special treatment.

Conclusion: These results indicated that NBP and DXM combined with HBO for the treatment of DEACMP can significantly improve the cognitive and motor functions of patients and is very safe.

Keywords: butylphthalide, NBP, dexamethasone, DXM, hyperbaric oxygen, HBO, DEACMP, carbon monoxide poisoning

\section{Introduction}

$\mathrm{CO}$ is an odourless, colourless, and tasteless gas that is highly difficult to detect when it is escaping. Currently, $\mathrm{CO}$ poisoning is a common health problem in many countries, ${ }^{1}$ primarily because of its severe clinical effects and high toxicological morbidity and mortality. ${ }^{2} \mathrm{CO}$ affects nearly all tissues and organs, but the toxidromes lack clinical specificity and are often overlooked and misdiagnosed. Symptoms of mild acute CO poisoning include headache, lightheadedness, confusion, vertigo, and flu-like effects; exposure to $\mathrm{CO}$ for long periods can cause severe toxicity in the cardiovascular systems 
and the central nervous system. In addition, acute neurological sequelae (loss of consciousness, coma, and death) may be observed in people recovering from $\mathrm{CO}$ poisoning. In fact, acute brain injury and DEACMP are the most common neurological complications. ${ }^{3,4} \mathrm{CO}$ poisoning can cause neurological, psychiatric, behavioural and cognitive disorders, including cortical blindness, parkinsonism, amnesia, personality changes, dementia, and mental disorders such as anxiety and depression. Moreover, dementia is a progressive and incurable disease. In patients with advanced dementia, the year before death is characterized by a series of persistent severe disabilities, including profound memory impairment, such as inability to recognize family members, minimal verbal abilities, inability to independently ambulate, inability to perform activities of daily living, and urinary or faecal incontinence, ${ }^{5,6}$ which impose a heavy economic and emotional burden on families and society.

Currently, the specific mechanisms of DEACMP are still unclear. A number of factors have been found to be associated with the development of DEACMP, such as ischaemia, hypoxia, immune dysfunction, cytotoxic injury, neurotransmitter dysregulation, and reperfusion injury. ${ }^{7-9}$ HBO was often recommended by previous studies to treat patients with acute $\mathrm{CO}$ poisoning. ${ }^{10,11}$ Researchers have also reported that $\mathrm{HBO}$ has significant effects on DEACMP. ${ }^{12-14}$ It is difficult to control the progression of DEACMP in a timely and effective manner only using HBO. Therefore, the exploration of more effective drugs is still the focus of current research.

The main active component of NBP is dl3-N-butylphthalide, which was originally isolated from celery seeds. NBP is a lipid-soluble drug with good bloodbrain barrier permeability. ${ }^{15}$ NBP soft capsules are the only new drug to cure cerebral arterial thrombosis in cerebrovascular disease and were approved by China Food and Drug Administration (CFDA) in 2002. Therefore, NBP became the third original new drug with proprietary intellectual property rights in China after artemisinin and bicyclol. In animal models of cerebral apoplexy, NBP was mainly used for inhibiting platelet aggregation, reducing thrombosis, improving microcirculation, reducing the volume of cerebral infarction, resisting oxidative stress, protecting mitochondrial function, reducing neuronal apoptosis, alleviating inflammatory reaction, mediating autophagy of nerve cells, promoting neurogenesis and targeting in several pathophysiological mechanisms, which are mainly reflected in its obvious neuroprotective effect. ${ }^{16-26}$ After further studies on its application, it was found that the beneficial effects of NBP are beyond those of apoplexy. Especially in neurodegenerative disease fields, it was found that NBP and its ramifications have unique function and effects in $\mathrm{AD}$, vascular dementia (VaD), PD, ALS and DEACMP treatment. ${ }^{27-30}$ In addition, relevant clinical studies have shown that short-term NBP and HBO have a good clinical effect on the recovery of the cognitive function of DEACMP patients. ${ }^{31,32}$ Relevant studies have also shown that DXM and HBO also have a good clinical effect on the recovery of cognitive function of DEACMP patients. ${ }^{33}$

Therefore, we conducted a study to investigate the effects of NBP and DXM combined with HBO on the treatment of mental symptoms and cognitive dysfunction in DEACMP patients at 90 days and to test the effectiveness and safety of this treatment. This study provides a basis for the clinical application of NBP and DXM for $\mathrm{CO}$ poisoning.

\section{Patients and Methods \\ Recruited Patients}

The Institutional Review Board of Heze municipal hospital reviewed and approved the purpose and design of our work. Informed consent in written or oral form was formally obtained from all participants and was conducted in accordance with the Declaration of Helsinki. If any patients have passed away, consent was required from the patient's next of kin. This clinical trial was performed between October 2013 and October 2018.

\section{Experimental Methods}

In this single-centre trial and retrospective cohort study, the included patients were assigned into two groups: the combined therapy and control groups. Conventional treatments, including using antibiotics, performing sputum suction, reducing or avoiding respiratory tract infection, maintaining unobstructed airways, maintaining stable blood pressure and blood glucose, regulating water electrolytes, and preventing urinary tract infection, were provided to all patients.

Patients meeting the following criteria were included: (1) patients with a history of $\mathrm{CO}$ exposure; (2) patients with a Mini-Mental State Examination (MMSE) score of no more than $24 ;{ }^{34}$ (3) patients with clinical symptoms of DEACMP occurring after 2-60 days of a latent period without any clinical manifestation of acute CO poisoning; (4) patients with neuroimaging evidence of demyelination lesions of 
bilateral cerebral white matter in brain MRI; and (5) patients who provided written or oral informed consent and completed all of the treatment programme and a follow-up plan.

The exclusion criteria were as follows: history of severe allergy to NBP or DXM; patients who had chronic hepatic or renal insufficiency; intellectual impairment; stroke; systemic infection or severe local infection; coagulation disorders; HIV, hepatitis B virus (HBV)/C virus (HCV); syphilis; genetic disorders or pregnancy.

Patients in the two groups were placed in the oxygen chamber with $0.25 \mathrm{MPa}$ absolute pressure for $80 \mathrm{~min} /$ day. The patients in the combined therapy group received NBP, DXM and HBO. Then, $100 \mathrm{~mL}$ of an NBP injection was given twice daily in the subsequent 14 days, and 76 days after hospital discharge, $200 \mathrm{mg}$ NBP soft capsules were intravenously infused three times daily. The DXM treatment was provided for 5 days/week for 4 weeks. The doses of DXM mainly referred to the previous study that used DXM to treat CO-poisoned patients. ${ }^{35}$ Researchers followed up and assessed the patients at 1 month, 3 months, and 1 year.

\section{Outcome Assessment Primary Outcome}

The cognition and movement changes of patients were evaluated by the Mini-Mental State Examination (MMSE), Montreal Cognitive Assessment (MoCA) scale and the Barthel index of activities of daily living (ADL) before and after the treatment at 1 month, 3 months, and 1 year. The total ADL score was set to 100 , and the higher the score, the better the abilities of daily life of the patients. Moreover, the total MMSE and MoCA scores were both set to 30 , and higher scores indicated stronger cognitive ability of the patients. Combined with the application of MoCA, MMSE can initially screen for various cognitive disorders, improving the detection rate of cognitive impairment, increasing the sensitivity and specificity, and reducing the false-positive rate and false-negative rate.

\section{Secondary Results}

The potential adverse events were also recorded and analysed to assess the acceptability of the combined application of NBP, DXM and HBO therapy.

\section{Statistical Analysis}

All statistical analyses were performed using SPSS 22.0 statistical software, and all the continuous variables are expressed as the mean \pm standard deviation. Student's $t$-test and the chi-squared test were applied to investigate the differences between the two groups in regard to demographic and baseline clinical variables. We performed an analysis of covariance (ANCOVA) to assess the effect of the treatment modalities on the posttreatment ADL, MoCA and MMSE scores. All procedures were 2-tailed, and a value of $\mathrm{P}<0.05$ was considered statistically significant for all tests.

\section{Results}

\section{Demographic Characteristics}

A total of 179 patients with DEACMP were recruited at the start of the study. During the treatment, 5 cases in the control group and 3 cases in the combined treatment group were lost to follow-up. Finally, 171 patients completed the entire treatment. There were 80 and 91 patients in the control and combined therapy groups, respectively. There were no significant differences in any baseline data, such as age, sex, educational level, profession, body mass index (BMI), CO exposure time, carboxyhemoglobin levels, latent phase, coma time, Glasgow coma scale, or Acute Physiology and Chronic Health Evaluation II (APACHE II). The detailed information is shown in Table 1 .

\section{Treatment Efficacy}

At 1 month, 3 months, and 1 year after the treatment, the MMSE scores were all significantly higher in the combined therapy group than in the control group (22.07 \pm 2.323 vs $20.45 \pm 3.405 ; 25.36 \pm 2.383$ vs $24.20 \pm 3.331$; $28.26 \pm 2.097$ vs $26.55 \pm 3.077$; all $\mathrm{P}<0.001)$. Moreover, the MoCA scores were all significantly higher in the combined therapy group than in the control group $(20.70$ \pm 2.783 vs $19.75 \pm 3.396 ; 23.80 \pm 2.642$ vs $22.70 \pm 3.262$; $27.05 \pm 2.297$ vs $5.75 \pm 3.440$; all $\mathrm{P}<0.05)$. In addition, compared with the control group, the ADL scores were also markedly increased in the combined therapy group at 1 month $(55.38 \pm 8.668$ vs $47.75 \pm 6.746), 3$ months $(69.23 \pm 11.178$ vs $57.00 \pm 8.479)$, and 1 year $(87.69 \pm$ 9.259 vs $78.25 \pm 12.855$ ) after the treatment (all $\mathrm{P}<0.05$ ). The detailed information is shown in Table 2 .

\section{Adverse Events}

There were no significant alterations in blood glucose, blood lipids, or liver and kidney function during the whole treatment session, partly because of the low dose and the short time of DXM use. In the combined therapy group, three patients experienced loss of appetite, three patients reported mild headache, and two patients had 
Table I Baseline Characteristics and Clinical Data of the Included Patients

\begin{tabular}{|c|c|c|c|c|}
\hline & Combined-Therapy Group $(n=91)$ & Control Group $(n=80)$ & $t / x 2$ & P-value \\
\hline Age, y & $59.64 \pm 12.284$ & $58.59 \pm 13.632$ & 0.530 & 0.111 \\
\hline Range & $18-80$ & $18-80$ & & \\
\hline Female, n (\%) & $42(46.2 \%)$ & $31(38.8 \%)$ & 0.954 & 0.355 \\
\hline BMI $\left(\mathrm{kg} / \mathrm{m}^{2}\right)$ & $21.96 \pm 2.616$ & $22.50 \pm 2.890$ & -1.292 & 0.228 \\
\hline Education level $(y)$ & $9.96 \pm 2.616$ & $9.95 \pm 2.575$ & 0.015 & 0.838 \\
\hline Mental labour, n(\%) & $29(31.9 \%)$ & $29(36.3 \%)$ & 0.365 & 0.628 \\
\hline $\mathrm{CO}$ exposure time $(\mathrm{h})$ & $9.64 \pm 4.974$ & $10.63 \pm 4.824$ & -1.314 & 0.613 \\
\hline $\mathrm{COHb}$ levels (\%) & $27.07 \pm 7.916$ & $25.38 \pm 7.445$ & 1.433 & 0.486 \\
\hline Latent phase $(\mathrm{d})$ & $14.67 \pm 5.385$ & $13.63 \pm 4.824$ & 1.329 & 0.218 \\
\hline Coma time $(\mathrm{h})$ & $6.87 \pm 3.212$ & $6.50 \pm 2.890$ & 0.783 & 0.235 \\
\hline Glasgow Coma Scale & $10.79 \pm 1.710$ & $10.94 \pm 1.625$ & 0.571 & 0.569 \\
\hline APACHE II & $14.02 \pm 5.40$ & $14.39 \pm 5.089$ & 0.442 & 0.659 \\
\hline Current/previous smoking & $49(53.8 \%)$ & $39(48.8 \%)$ & 0.443 & 0.542 \\
\hline Hypertension & $38(41.8 \%)$ & $38(47.5 \%)$ & 0.568 & 0.538 \\
\hline Hypercholesterolemia & $50(54.9 \%)$ & $47(58.8 \%)$ & 0.645 & 0.251 \\
\hline Diabetes & $12(13.2 \%)$ & $16(20.0 \%)$ & 1.443 & 0.301 \\
\hline Coronary heart disease & $15(16.5 \%)$ & $10(12.5 \%)$ & 0.541 & 0.520 \\
\hline
\end{tabular}

Notes: Data presented as mean \pm standard deviation or ratio. $t / x 2$ means the value is either Student's $t$-test or the chi-squared test.

Abbreviations: BMI, body mass index; $\mathrm{CO}$, carbon monoxide; h, hours; COHb, carboxyhemoglobin; APACHE, Acute Physiology and Chronic Health Evaluation.

Table 2 Comparison of MMSE, MoCA and ADL at Different Time Points Before and After Treatment in Two Groups

\begin{tabular}{|l|l|l|l|}
\hline & Combined-Therapy Group (N =9 I) & Control Group (N=80) & P-value \\
\hline The MMSE Scores of the 2 Groups & & & \\
Before treatment & $16.49 \pm 3.871$ & $15.90 \pm 4.160$ & 0.334 \\
I month after treatment & $22.07 \pm 2.323$ & $20.45 \pm 3.405$ & 0.000 \\
3 months after treatment & $25.36 \pm 2.383$ & $24.20 \pm 3.331$ & 0.000 \\
I year after treatment & $28.26 \pm 2.097$ & $26.55 \pm 3.077$ & 0.000 \\
\hline The MoCA Scores of the 2 Groups & & & \\
Before treatment & $14.95 \pm 3.837$ & $15.21 \pm 3.964$ & 0.987 \\
I month after treatment & $20.70 \pm 2.783$ & $19.75 \pm 3.396$ & 0.002 \\
3 months after treatment & $23.80 \pm 2.642$ & $22.70 \pm 3.262$ & 0.002 \\
I year after treatment & $27.05 \pm 2.297$ & $25.75 \pm 3.440$ & 0.000 \\
\hline The ADL Scores of the 2 Groups & & & \\
Before treatment & $12.42 \pm 5.596$ & $13.00 \pm 5.131$ & 0.214 \\
I month after treatment & $55.38 \pm 8.668$ & $47.75 \pm 6.746$ & 0.001 \\
3 months after treatment & $69.23 \pm I I .178$ & $57.00 \pm 8.479$ & 0.003 \\
I year after treatment & $87.69 \pm 9.259$ & $78.25 \pm 12.855$ & 0.000 \\
\hline
\end{tabular}

minor skin irritations. In the control group, two patients had loss of appetite, and two patients reported mild headache. However, these patients recovered by themselves and needed no additional medications or special treatment.

\section{Discussion}

In our study, we found that HBO therapy as a monotherapy could significantly increase the MMSE scores and decrease the NIHSS scores of patients with DEACMP after acutephase treatment. Moreover, we found that the addition of NBP and DXM could significantly improve cognitive function in patients with DEACMP treated by HBO. In addition, the ADL score increased significantly in the experimental group, suggesting that the addition of NBP and DXM to the course of disease management may have a better effect in protecting and restoring nervous function. In addition, the 
addition of NBP and DXM did not significantly increase the incidence of adverse events. Based on the above results, NBP and DXM combined with $\mathrm{HBO}$ in the treatment of DEACMP can achieve better clinical efficacy, and it is expected to become a potential new treatment method for DEACMP patients but requires further exploration.

DEACMP is the most common neurological complication after acute $\mathrm{CO}$ poisoning and is also a serious disease worldwide. The main manifestations are advanced intelligence loss, mental behaviour abnormalities, and dyskinesia, which seriously affect the quality of life of patients. The language, memory, and visual function of DEACMP patients rapidly decline in a very short term, and personality abnormalities and other cognitive functions (including judgement, generalization, and multi-ability in analysing and solving problems) appeared at a later stage in severe cases and were often accompanied by emotional disorders and pyramidal and extrapyramidal system dysfunction. At present, the detailed pathogenesis of DEACMP is not clear. Convincing evidence suggests that the main pathological mechanism of DEACMP may be delayed neuronal death in the hippocampal CA1 subarea $^{36}$ and extensive demyelinating lesions of white matter in the brain tissue. ${ }^{37}$ The pathogenesis of delayed neuronal death in the hippocampal CA1 subarea has been proven, including inflammation, increased excitatory amino acids, abnormal neurotransmitters, mitochondrial function inhibition, oxidative stress in mitochondria, apoptosis, lipid peroxidation and adaptive immune response. ${ }^{38-40}$ Gorman et al reported that after $\mathrm{CO}$ poisoning, ischaemia and hypoxia, oxygen-free radicals and peroxides in brain cells increased significantly, and lipid peroxidation in the cell membrane was enhanced, leading to demyelination of nerve cells and secondary neuronal cell death. ${ }^{41,42}$ Other researchers reported that DEACMP is immune-mediated and that delayed synergisticmediated neuropathology is associated with an adaptive immune response to chemically modified myelin basic protein. ${ }^{7}$ Most scholars believe that $\mathrm{CO}$ poisoning might lead to structural and functional changes of myelin basic proteins, the abnormal expression of myelin-derived axonal growth inhibitors (such as Nogo, NgR, and Ogmp), and the imbalanced expression of cytokines and inflammatory molecules in the central nervous system, which may be an important factor of generalized demyelination lesions in white matter of brain tissue. ${ }^{43,44}$ In addition, Ischiropoulos et al reported that $\mathrm{CO}$ poisoning altered nitric oxide (NO) levels and NO synthase activity, suggesting that NO may be involved in brain tissue damage. ${ }^{45}$ DEACMP occurs due to the interaction of many factors.
NBP is a new type of anti-cerebral ischaemia drug. Its main active ingredient is dl-3-N-butylphthalide, which is a fat-soluble substance that can pass through the bloodbrain barrier. The mechanism of its effect on DEACMP may be correlated with its unique pharmacological effects: (1) selectively inhibiting the physiological and pathological process mediated by arachidonic acid and its metabolites, reversing microvasospasm to inhibit platelet aggregation and reduce thrombosis; (2) directly acting on the mitochondria in nerve cells of cerebral ischaemia areas, enhancing the fluidity of the mitochondrial membrane, improving the activity of complex IV enzyme in the respiratory chain, enhancing the activity of antioxidant enzymes, reducing the generation of oxygen-free radicals, and thereby increasing the content of ATP and phosphocreatine in brain cells; (3) maintaining the complete structure of microvessels, increasing the number of microvessels, promoting collateral circulation in cerebral ischaemia areas, improving microcirculation, and significantly increasing regional cerebral blood flow; (4) inhibiting the release of glutamate, reducing the intracellular calcium concentration, resisting oxidative stress, and thereby alleviating the inflammatory response and cell apoptosis after cerebral ischaemia. Therefore, NBP reveals a significant neuroprotective effect, alleviating nerve damage and reducing the disability or fatality rates in patients with DEACMP; and (5) NBP can promote axon and synapse formation and nerve regeneration in the subventricular area after cerebral ischaemia and hypoxia; upregulate TREK-1 (TWIK-related $\mathrm{K}^{+}$channel 1, TREK-1) in the biforamen area; affect the proliferation of neural stem cells and astrocytes, ${ }^{46}$ significantly promote the proliferation of hippocampal cells after stroke; improve the survival rate of new cells; regulate the differentiation of new cells into mature neurons; improve the memory function of specific nerve cells; and effectively reverse or improve cognitive impairment.

At present, we have found that DXM can alleviate inflammation by protecting the myelin sheath from inflammation. Atalay et $\mathrm{al}^{47}$ reported that DXM might affect brain tissue lipid peroxidation while treating COpoisoned rats. Another animal study $^{48}$ indicated that early DXM treatment might have a positive effect on preventing DEACMP after 18 days of treatment. $\mathrm{HBO}$ therapy is beneficial to the recovery of damaged brain cells by increasing blood oxygen levels in brain tissue. It is suggested that NBP and DXM combined with HBO are effective for the treatment of DEACMP.

The limitations of our study are (1) its retrospective design; (2) its non-random nature; (3) the sample size is 
relatively small; (4) all patients were from the same city; and (5) only the short-term efficacy of the combination of NBP, DXM and HBO was examined. Therefore, further studies are needed to confirm the efficacy and safety of NBP and DXM combined with HBO in the treatment of DEACMP by prospective, randomized, multicentre, large sample studies with long-term longitudinal follow-up.

\section{Conclusion}

Our results indicated that NBP and DXM combined with HBO in the treatment of DEACMP may improve the cognitive and motor functions of patients without significant side effects. Our study should be viewed as a potential new therapy.

\section{Acknowledgment}

Sincere gratitude is extended to the nurses in our department and participants for their efforts and cooperation.

\section{Disclosure}

The authors report no conflicts of interest in this work.

\section{References}

1. Centers for disease control and prevention(CDC). Carbon monoxide exposures-United States, 2000-2009. MMWR. 2011;60 (30):1014-1017.

2. Kalay N, Ozdogru I, Cetinkaya Y, et al. Cardiovascular effects of carbon monoxide poisoning. Am J Cardiol. 2007;99(3):322-324. doi:10.1016/j.amjcard.2006.08.030

3. Geraldo AF, Silva C, Neutel D, Neto LL, Albuquerque L. Delayed leukoencephalopathy after acute carbon monoxide intoxication. J Radiol Case Rep. 2014;8(5):1-8. doi:10.3941/jrcr.v8i5.1721

4. Goldstein M. Carbon monoxide Poisoning. J Emerg Nurs. 2008;34 (6):538-542. doi:10.1016/j.jen.2007.11.014

5. Gill TM, Gahbauer EA, Han L, et al. Trajectories of disability in the last year of life. $N$ Engl $J$ Med. 2010;362(13):1173-1180. doi:10.1056/NEJMoa0909087

6. Mitchell SL, Teno JM, Kiely DK, et al. The clinical course of advanced dementia. $N$ Engl J Med. 2009;361(16):1529-1538. doi:10.1056/NEJMoa0902234

7. Thom SR, Bhopale VM, Fisher D, et al. Delayed neuropathology after carbon monoxide poisoning is immune-mediated. Proc Natl Acad Sci USA. 2004;101(37):13660-13665. doi:10.1073/ pnas.0405642101

8. Thom SR, Bhopale VM, Fisher D, Zhang J, Gimotty P. Delayed neuropathology after carbon monoxide poisoning is immune-mediated. Proc Natl Acad Sci USA. 2004;101 (37):13660-13665. doi:10.1073/pnas.0405642101

9. Guzman JA. carbon monoxide poisoning. Crit Care Clin. 2012;28 (4):537-548. doi:10.1016/j.ccc.2012.07.007

10. Weaver LK, Hopkins RO, Chan KJ, et al. Hyperbaric oxygen for acute carbon monoxide poisoning. $N$ Engl J Med. 2002;347 (14):1057-1067. doi:10.1056/NEJMoa013121

11. Annane D, Chadda K, Gajdos P, et al. Hyperbaric oxygen therapy for acute domestic carbon monoxide poisoning: two randomized controlled trials. Intensive Care Med. 2011;37(3):486-492. doi:10.1007/ s00134-010-2093-0
12. Weaver LK, Valentine KJ, Hopkins RO. Carbon monoxide poisoning: risk factors for cognitive sequelae and the role of hyperbaric oxygen. Am J Respir Crit Care Med. 2007;176(5):491-497. doi:10.1164/ rccm.200701-026OC

13. Weaver LK. Hyperbaric oxygen therapy for carbon monoxide poisoning. Undersea Hyperb Med. 2014;41(4):339-354.

14. Mao M, Rao P, Mou X, et al. Clinical observation on delayed encephalopathy after carbon monoxide poisoning treated with acupuncture to restore consciousness combined with hyperbaric oxygen treatment. Zhongguo Zhen Jiu. 2015;35(3):213-216.

15. Diao XX, Zhong K, XL L, et al. Isomer-selective distribution of 3-nbutylphthalide (NBP) hydroxylated metabolites, 3-hydroxy-NBP and 10-hydroxy-NBP, across the rat blood-brain barrier. Acta Pharmacol Sin. 2015;36(12):1520-1527. doi:10.1038/aps.2015.64

16. Peng Y, Xing C, Xu S, et al. L-3-n-butylphthalide improves cognitive impairment induced by intracerebroventricular infusion of amyloid- $\beta$ peptide in rats. Eur J Pharmacol. 2009;621(1-3):38-45. doi:10.1016/ j.ejphar.2009.08.036

17. Xu J, Huai Y, Meng N, et al. L-3-n-Butylphthalide activates Akt/ mTOR signaling, inhibits neuronal apoptosis and autophagy and improves cognitive impairment in mice with repeated cerebral ischemia-reperfusion injury. Neurochem Res. 2017;42 (10):2968-2981. doi:10.1007/s11064-017-2328-3

18. Zhao Y, Lee JH, Chen D, et al. DL-3-n-butylphthalide induced neuroprotection, regenerative repair, functional recovery and psychological benefits following traumatic brain injury in mice. Neurochem Int. 2017;111:82-92. doi:10.1016/j.neuint.2017.03.017

19. Abdoulaye IA, Guo YJ. A review of recent advances in neuroprotective potential of 3-n-Butylphthalide and its derivatives. Biomed Res Int. 2016;2016:5012341. doi:10.1155/2016/5012341

20. Chen Y, Wu T, Li H, et al. Dl-3-n-Butylphthalide exerts dopaminergic neuroprotection through inhibition of neuroinflammation. Front Aging Neurosci. 2019;11:44.

21. Fitzpatrick T, Gocan S, Wang CQ, et al. How do neurologists diagnose transient ischemic attack: a systematic review. Int $J$ Stroke. 2019;14(2):115-124. doi:10.1177/1747493018816430

22. Li F, Ma Q, Zhao H, et al. L-3-n-Butylphthalide reduces ischemic stroke injury and increases M2 microglial polarization. Metab Brain Dis. 2018;33(6):1995-2003. doi:10.1007/s11011-018-0307-2

23. Ye X, Rong Z, Li Y, et al. Protective role of L-3-n-Butylphthalide in cognitive function and dysthymic disorders in mouse with chronic epilepsy. Front Pharmacol. 2018;9:734. doi:10.3389/fphar.2018.0 0734

24. Yin W, Lan L, Huang Z, et al. Discovery of a ring-opened derivative of 3-n-butylphthalide bearing $\mathrm{NO} / \mathrm{H} 2 \mathrm{~S}$-donating moieties as a potential anti-ischemic stroke agent. Eur J Med Chem. 2016;115:369-380. doi:10.1016/j.ejmech.2016.03.044

25. Zhou PT, Wang LP, Qu MJ, et al. Dl-3-N-butylphthalide promotes angiogenesis and upregulates sonic hedgehog expression after cerebral ischemia in rats. CNS Neurosci Ther. 2019;25(6):748-758. doi:10.1111/cns.2019.25.issue-6

26. Wang L, Wang X, Li T, Zhang Y, Ji H. 8e protects against acute cerebral ischemia by inhibition of PI3K gamma-mediated superoxide generation in microglia. Molecules. 2018;23:11.

27. Peng Y, Sun J, Hon S, et al. Lemere, L-3-n-butylphthalide improves cognitive impairment and reduces amyloid- $\beta$ in a transgenic model of alzheimer's disease. $J$ Neurosci. 2010;30(24):8180-8189. doi:10.1523/JNEUROSCI.0340-10.2010

28. Feng X, Peng Y, Liu M, Cui L. DL-3-n-butylphthalide extends survival by attenuating glial activation in a mouse model of amyotrophic lateral sclerosis. Neuropharmacology. 2012;62(2):1004-1010. doi:10.1016/j.neuropharm.2011.10.009

29. Xiong N, Huang J, Chen C, et al. Dl-3-n-butylphthalide, a natural antioxidant, protects dopamine neurons in rotenone models for Parkinson's disease. Neurobiol Aging. 2012;33(8):1777-1791. doi:10.1016/j.neurobiolaging.2011.03.007 
30. Li Q, Cheng Y, Bi M, et al. Effects of N-butylphthalide on the activation of Keap1/Nrf-2 signal pathway in rats after carbon monoxide poisoning. Environ Toxicol Pharmacol. 2015;40(1):22-29. doi:10.1016/j.etap.2015.05.009

31. Xiang W, Xue H, Wang B, et al. Efficacy of N-Butylphthalide and hyperbaric oxygen therapy on cognitive dysfunction in patients with delayed encephalopathy after acute carbon monoxide poisoning. Med Sci Monit. 2017;23:1501-1506. doi:10.12659/MSM.899499

32. Wang H, Li Y, Wu Q, Xu C, Liu Q. Combination of butylphthalide with umbilical mesenchymal stem cells for the treatment of delayed encephalopathy after carbon monoxide poisoning. Medicine (Baltimore). 2016;95(49):e5412. doi:10.1097/MD.0000000000005412

33. Xiang W, Xue H, Wang B, et al. Combined application of dexamethasone and hyperbaric oxygen therapy yields better efficacy for patients with delayed encephalopathy after acute carbon monoxide poisoning. Drug Des Devel Ther. 2017;11:513-519. doi:10.2147/ DDDT.S126569

34. Shu AH, Wang Q, Chen XB. Effect of different depths of anesthesia on postoperative cognitive function in laparoscopic patients: a randomized clinical trial. Curr Med Res Opin. 2015;31 (10):1883-1887. doi:10.1185/03007995.2015.1075968

35. Ma XY. Clinical study of large dose of dexamethasone combined with hyperbaric oxygen in treatment of acute carbon monoxide poisoning. Med Innov China. 2013;10(23):045-047.

36. Berl WG, Halpin B. Human fatalities from unwanted fires. Johns Hopkins University, applied physics laboratory, fire problems program. APL/JHW FPP. Baltimore, MD;1978.p.TR 37.

37. Kim JH, Chang KH, Song IC, et al. Delayed encephalopathy of acute carbon monoxide intoxication: diffusivity of cerebral white matter lesions. AJNR Am J Neuroradiol. 2003;24(8):1592-1597.

38. Raub JA, Mathieu-Nolf M, Hampson NB, Thom SR. Carbon monoxide poisoning - a public health perspective. Toxicology. 2000;145 (1):1-14. doi:10.1016/S0300-483X(99)00217-6

39. Penney D, Benignus V, Kephalopoulos S, Kotzias D, Kleinman M, Verrier A. Carbon monoxide. In: WHO guidelines for indoor air quality: selected pollutants. 2010. Available from: http://www.ncbi. nlm.nih.gov/books/NBK138710/. Accessed August 23, 2016
40. Hampson NB, Piantadosi CA, Thom SR, Weaver LK. Practice recommendations in the diagnosis, management, and prevention of carbon monoxide poisoning. Am J Respir Crit Care Med. 2012;186 (11):1095-1101. doi:10.1164/rccm.201207-1284CI

41. Gorman D, Drewry A, Huang YL, Sames C. The clinical toxicology of carbon monoxide. Toxicol. 2003;187(1):25-38. doi:10.1016/ S0300-483X(03)00005-2

42. Nabeshima T, Katoh A, Ishim H, et al. Carbon monoxide-induced delayed amnesia, delayed neuronal death and change in acetylcholine concentration in mice. Pharmacol Exp Ther. 1991;256:378-384.

43. Li Q, Cheng Y, Bi MJ, et al. Effects of N-Butylphthalide on the expressions of $\mathrm{Nogo} / \mathrm{NgR}$ in rat brain tissue after carbon monoxide poisoning. Environ Toxicol Pharmacol. 2015;39(2):953-961. doi:10.1016/j.etap.2015.02.013

44. Yang Y, Liu Y, Wei P, et al. Silencing Nogo-A promotes functional recovery in demyelinating disease. Ann Neurol. 2010;67(4):498-507. doi:10.1002/ana.21935

45. Ischiropoulos H, Beers MF, Ohnishi ST, et al. Nitric oxide production and perivascular nitration in brain after carbon monoxide poisoning in the rat. J Clin Invest. 1996;97(10):2260-2267. doi:10.1172/ JCI118667

46. Wang M, Song J, Xiao W, et al. Changes in lipid-sensitive two-pore domain potassium channel TREK-1 expression and its involvement in astrogliosis following cerebral ischemia in rats. $J$ Mol Neurosci. 2012;46(2):384-392. doi:10.1007/s12031-011-9598-Z

47. Atalay H, Aybek H, Koseoglu M, et al. The effects of amifostine and dexamethasone on brain tissue lipid peroxidation during oxygen treatment of carbon monoxide-poisoned rats. Adv Ther. 2006;23 (2):332-341. doi:10.1007/BF02850138

48. Li Q, Song JJ, Zhang HY, Fu K, Lan HB, Deng Y. Dexamethasone therapy for preventing delayed encephalopathy after carbon monoxide poisoning. Biotech Histochem. 2015;90(8):561-567. doi:10.3109/ 10520295.2015 .1019565
Drug Design, Development and Therapy

\section{Publish your work in this journal}

Drug Design, Development and Therapy is an international, peerreviewed open-access journal that spans the spectrum of drug design and development through to clinical applications. Clinical outcomes, patient safety, and programs for the development and effective, safe, and sustained use of medicines are a feature of the journal, which has also

\section{Dovepress}

been accepted for indexing on PubMed Central. The manuscript management system is completely online and includes a very quick and fair peer-review system, which is all easy to use. Visit http://www. dovepress.com/testimonials.php to read real quotes from published authors. 